$11-1-2005$

\title{
Entropic Characterization of Mixing in Microchannels
}

\author{
Marco Camesasca \\ Case Western Reserve University \\ Ica Manas Zloczower \\ Case Western Reserve University, ica.manas-zloczower@case.edu \\ Miron Kaufman \\ Cleveland State University, m.kaufman@csuohio.edu
}

Follow this and additional works at: https://engagedscholarship.csuohio.edu/sciphysics_facpub

Part of the Physics Commons

How does access to this work benefit you? Let us know!

\section{Publisher's Statement}

This is an author-created, un-copyedited version of an article accepted for publication in Journal of Micromechanics and Microengineering. The publisher is not responsible for any errors or omissions in this version of the manuscript or any version derived from it. The Version of Record is available online at http://iopscience.iop.org/article/10.1088/0960-1317/15/11/007/ meta;jsessionid=25E1916414D8541DEA5C68BD411381E2.c1.iopscience.cld.iop.org

\section{Repository Citation}

Camesasca, Marco; Zloczower, Ica Manas; and Kaufman, Miron, "Entropic Characterization of Mixing in Microchannels" (2005). Physics Faculty Publications. 236.

https://engagedscholarship.csuohio.edu/sciphysics_facpub/236

This Article is brought to you for free and open access by the Physics Department at EngagedScholarship@CSU. It has been accepted for inclusion in Physics Faculty Publications by an authorized administrator of EngagedScholarship@CSU. For more information, please contact library.es@csuohio.edu. 


\title{
Entropic characterization of mixing in microchannels
}

\author{
Marco Camesasca , Ica Manas-Zloczower and Miron Kaufman
}

\begin{abstract}
A methodology for rigorous mixing assessment in microchannels is presented. The analysis is based on numerical simulations of flow in different geometries coupled with mixing assessment using entropic measures. The results show enhanced mixing efficiency for the staggered herringbone micromixer by comparison with a mixer with straight diagonal ridges and a lack of mixing in a non-patterned channel. These results are in agreement with published experimental data.
\end{abstract}

\section{Introduction}

Microfluidic systems (lab-on-a-chip devices) are widely employed in microtechnological areas such as biology, medicine, chemistry, chemical engineering and biotechnology; applications vary from protein and DNA analysis, high-throughput screening, autonomic materials, sensors, chemical reactions, small quantities transfer $(1-100 \mathrm{nl})$ of fluids, fluidic-based computers, microelectromechanical systems (MEMS) [1-5]. These systems generally operate in a pressure-driven flow regime with no moving parts.

Micromixers are microfluidic systems where two or more fluids are mixed; these systems are used, for example, for controlling the dispersion of minor components in Poiseuille flows and for homogenization of solutions in chemical reactions [6]. Microfluidic devices generally operate in a laminar flow regime and consequently mixing is a difficult task [7]. The Reynolds number, $R e=u C / \mu(u$ is the average velocity of the fluid, $C$ is a characteristic crosssectional dimension and $\mu$ is the kinematic viscosity of the fluid) in such systems is generally far below the 2000-3000 range required for the onset of turbulence, primarily due to the very small characteristic dimensions. Moreover, the Peclet number, $P e=u C / D(D$ is the molecular diffusivity of the fluid) is generally high, indicating that diffusion is negligible by comparison to convection. This is particularly true in the case where mixing involves fluids containing large molecules for which diffusion is very slow [8]. Taking into account all of the above limitations the only viable route for achieving fast mixing in such systems is by manipulating the system geometry to force the fluids to stretch and fold and thereby to increase their interfacial area through the formation of striations of one fluid into the other [9]. System geometry is thus considered to hold the key of success in mixing at the microscale, and consequently patterned channels of various geometries have been designed and manufactured with the goal of increasing mixing efficiency [10].

Given that mixing in these types of systems critically depends on cross-sectional flow, all studies so far have been carried out focusing on the cross-sectional flow behavior and different methods/protocols have been used to characterize mixing. Experimentally, mixing evaluation has primarily relied on observing color or intensity variations of a dye as it is transported through the mixer $[11,12]$ or studying the interface configuration between two fluids, one of which is fluorescent, by employing confocal fluorescence microscopy [13]. To evaluate numerically the performance of different mixers, virtual particles are placed in the flow field and their trajectories are computed using a Lagrangian method. Interpreting the results of particle advection mostly relies upon recording and interpreting Poincare maps $[14,15]$ or the computation of Lyapunov exponents. In this case, the underlying assumption in the interpretation of the results is that mixing can be achieved efficiently only in chaotic flows [11, 14, 15]. In fact Lyapunov exponents are used primarily to evaluate the chaotic behavior of the system $[9,15,16]$. And whereas these criteria are intrinsically 
related to mixing, chaos is not a pre-requisite for mixing, and therefore a lack of chaos does not implicitly preclude system homogenization.

In this work we present an alternative way to characterize and quantify the degree of mixing in microchannels. We propose to use numerical simulations to study the flow patterns in channels with various geometries and to employ the conditional Shannon entropy to rigorously quantify the dynamics of mixing for two miscible fluids distinguished by color. Additionally, the proposed entropic analysis can be also carried out for data obtained in experiments, thus making it universally applicable for mixing evaluation.

\section{Background on statistical entropy}

The Shannon [17] entropy $S$ is the rigorous measure of mixing or a lack of information and it has been used in many different scientific areas [18-21]. It is uniquely determined from reasonable properties (Khinchin axioms [22]) that a measure of the lack of information or mixing must satisfy: (I) it depends on the probability distribution $p$ only; (II) the lowest entropy $(S=0)$ corresponds to one of the $p$ being 1 and the rest being zero (i.e., total information, perfect order, complete segregation); (III) the largest value for the entropy is achieved when all $p$ are equal to each other (i.e., the absence of any information, complete disorder, perfect mixing); and (IV) $S$ is additive over partitions of the outcomes. We have employed entropic measures for a variety of practical applications in polymer processing [23-25]. In this paper we extend this approach to the field of microfluidics.

The general expression of the Shannon entropy for a multi-component system with $C$ components distributed in a cross-sectional space divided into $M$ small equisized subdomains (bins) is:

$$
S=-\sum_{j=1}^{M} \sum_{c=1}^{C} p_{j, c} \ln p_{j, c},
$$

where $p_{j, c}$ is the joint probability that a particle is of component $c$ and is located in bin $j$. The total entropy is additive in the sense that it is equal to the sum of the conditional entropy $S_{\text {location }}$ (species) and the entropy of spatial distribution $S$ (location):

$$
\begin{gathered}
S_{\text {location }}(\text { species })=\sum_{j=1}^{M}\left[p_{j} S_{j} \text { (species) }\right] \\
S_{j} \text { (species) }=-\sum_{c=1}^{C}\left[p_{c / j} \ln p_{c / j}\right] .
\end{gathered}
$$

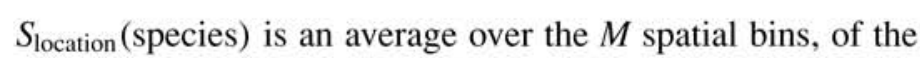
entropy of mixing the $C$ species conditional on bin location. In equations (2) and (3) $p_{j}$ is the probability that a particle is in bin $j$ irrespective of species and $p_{c / j}$ is the probability that a particle is of the type $c$ conditional on being in bin $j$. $S$ (location) is the entropy associated with the spatial distribution of particles irrespective of their species:

$$
S(\text { location })=-\sum_{j=1}^{M} p_{j} \ln p_{j} .
$$

In this work we analyze the mixing of two incompressible fluids $(C=2)$. Since the density at any location does not change in time, the entropy $S$ (location) is also constant at all times during the process. Thus we concentrate on $S_{\text {location }}$ (species) which measures the local quality of mixing of the two fluids averaged over space. Since $0 \leqslant$ $S_{\text {location }}$ (species) $\leqslant \ln C$, we normalize the species entropy conditional on locations $S_{\text {location }}$ (species) on $\ln C$ to get an index of mixing varying between 0 for perfect segregation and 1 for perfect mixing. The number of bins, $M$, controls the scale of observation and an analysis of the variation of entropy with the number of bins enables us to gauge mixing quality as a function of the scale of observation. Kang and Kwon [26] proposed a measure of mixing based on the information entropy, but used arbitrary weighing factors in the definition of their measure. Moreover, their proposed mixing measure is not independent of the initial conditions, nor does it unequivocally define a maximum degree of mixing.

\section{Numerical procedure}

In recent years, the finite element method (FEM) [27] has found increased use and wider acceptance in solving computational fluid dynamics (CFD) [28] problems. Nowadays the method is well established and routinely used for solving flow problems.

In our studies we employed FIDAP, a commercial CFDFEM package to solve the 3D steady-state, isothermal flow of a Newtonian fluid in the various geometries. Massless, non-interacting virtual tracers distinguished by color were randomly placed at the entrance of the flow channel and their position was followed along the channel length. A particle tracking algorithm (equation (5)) was employed to calculate the particle trajectories. In equation (5) $\mathbf{x}$ and $\mathbf{v}$ are, respectively, the position and velocity vectors, $t_{0}$ is the initial time and $t$ is the final time. The procedure is repeated until all the tracers exit the mixer:

$$
\mathbf{x}(t)=\mathbf{x}\left(t_{0}\right)+\int_{t_{0}}^{t} \mathbf{v}(t) \mathrm{d} t .
$$

The fourth-order Runge-Kutta method is employed to integrate numerically equation (5).

\section{Simulations}

We study the mixing capabilities of three different microsystems: a rectangular channel with no patterns on the walls, a rectangular channel with simple straight ridges on the bottom wall and a rectangular channel with asymmetric $\mathrm{V}$ ridges similar to the staggered herringbone mixer (SHM) [10]. The main difference between the last two geometries is the configuration of the patterns placed on the bottom of the channels: while for the straight diagonal ridge (SDR) geometry all ridges are equal and they are placed at an angle of $35^{\circ}$ with respect to the $x$-axis, the asymmetric $\mathrm{V}$ ridge (AVR) mixer has ridges with two arms asymmetric in length both forming an angle of $35^{\circ}$ with the $x$-axis. Every six ridges the geometry is inverted so that each six ridges form the mirror image of the previous six ones. Schematics of these two geometries are shown in figures 1 (SDR) and 2 (AVR) with portions of the actual meshes used in the 

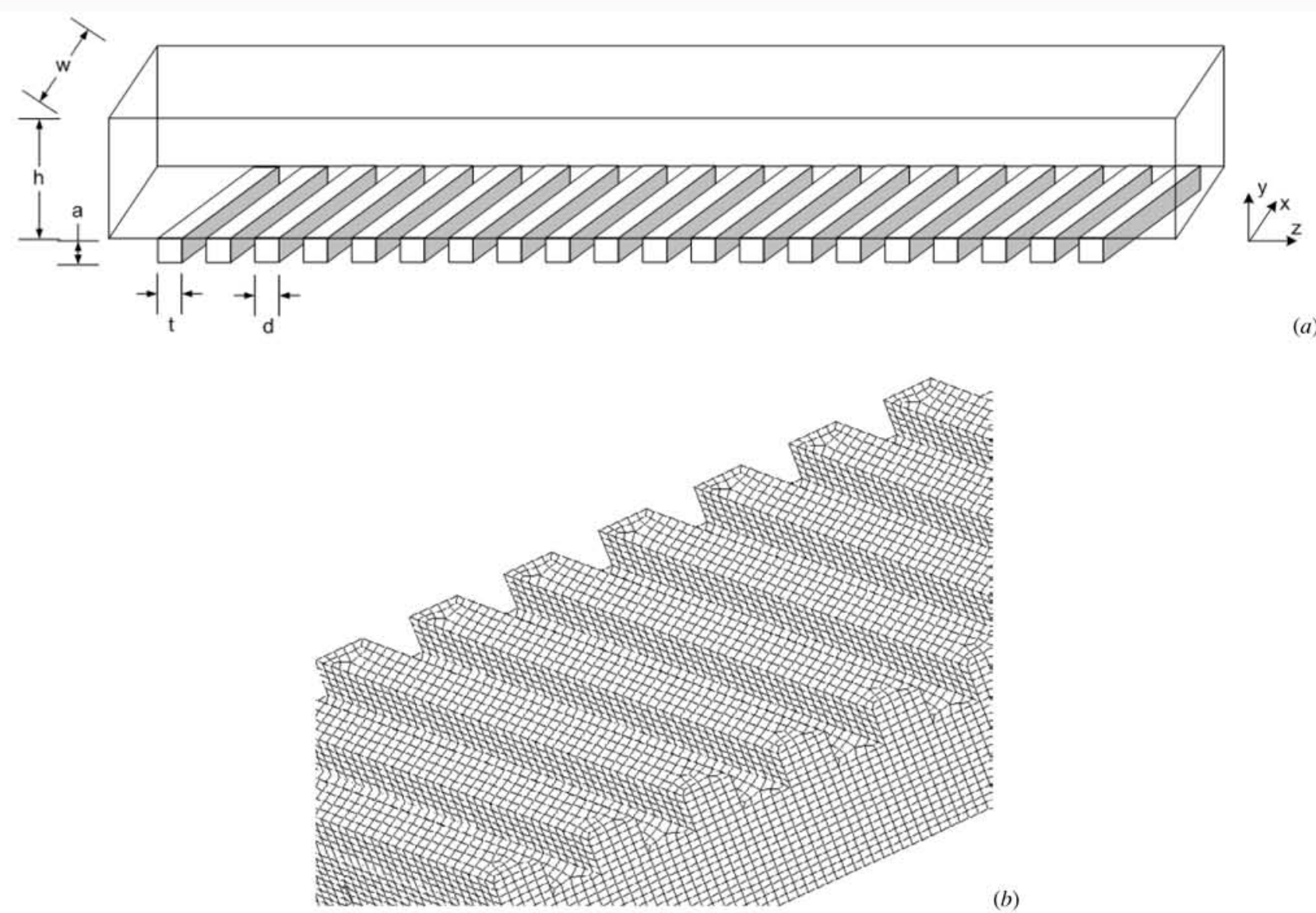

(a)

(b)

Figure 1. (a) Geometry of the channel with straight diagonal ridges (SDR). The height $h$ is $8 \times 10^{-5} \mathrm{~m}$, the width $w$ is $2 \times 10^{-4} \mathrm{~m}$, the ridge thickness $t$ and the ridge height $a$ are both $2 \times 10^{-5} \mathrm{~m}$; the minimum distance between two consecutive ridges $d$ is $2 \times 10^{-5} \mathrm{~m}$. The angle that each ridge forms with the $x$-axis is $35^{\circ}$. (b) Fraction of the mesh for the CFD simulation.

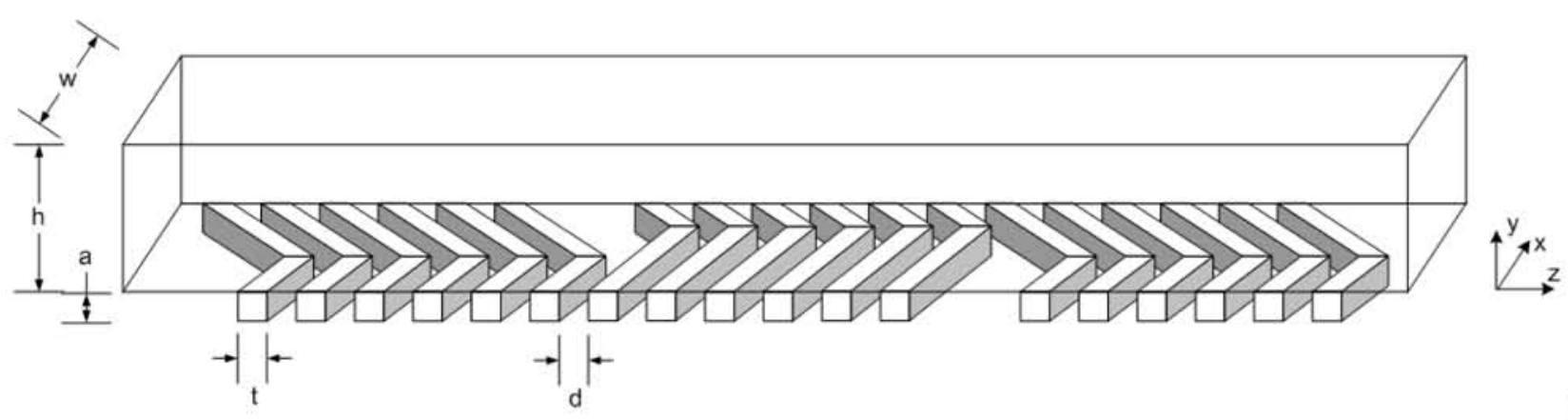

(a)

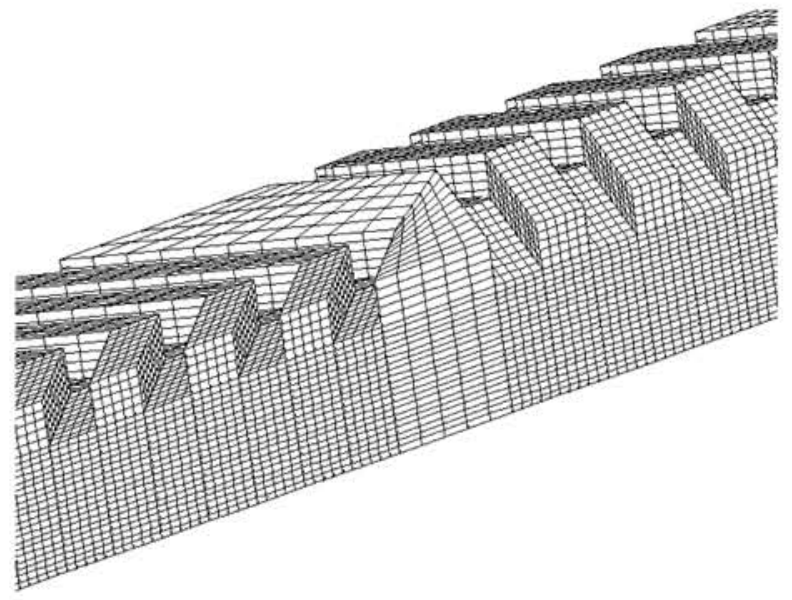

(b)

Figure 2. (a) Geometry of the channel with asymmetric V ridges (AVR). The height $h$ is $8 \times 10^{-5} \mathrm{~m}$, the width $w$ is $2 \times 10^{-4} \mathrm{~m}$, the ridge thickness $t$ and the ridge height $a$ are both $2 \times 10^{-5} \mathrm{~m}$; the minimum distance between two consecutive ridges $d$ is $2 \times 10^{-5} \mathrm{~m}$. The angle that each portion of the ridge forms with the $x$-axis is $35^{\circ}$. (b) Portion of the mesh for the CFD simulation. 


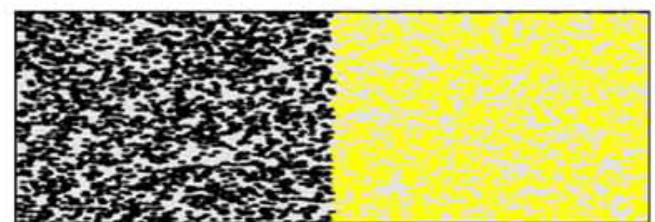

(a)

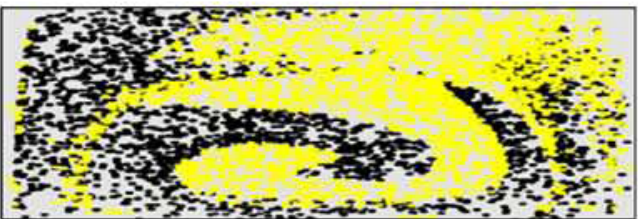

(c)

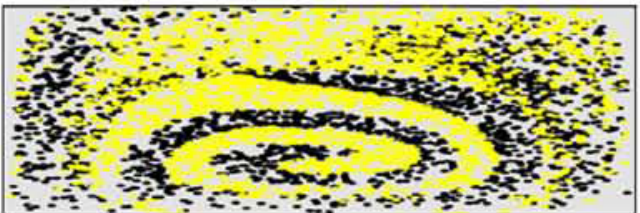

(e)

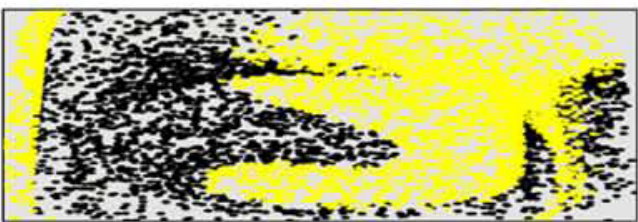

(b)

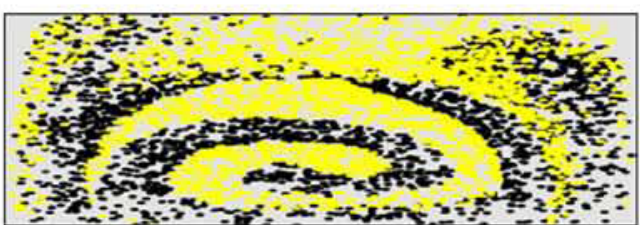

(d)

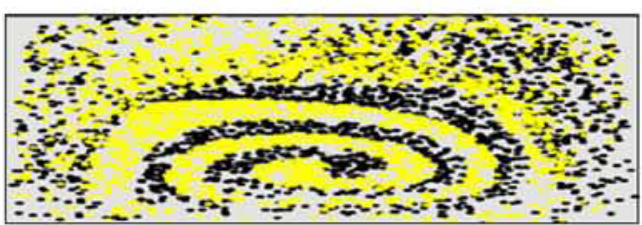

$(f)$

Figure 3. Evolution of mixing in the SDR micromixer. (a) Initial condition (the $x y$ cross-section at $z=0$ ); all consecutive images represent cross-sections of the system at $(b) z=1 \times 10^{-2} \mathrm{~m} ;(c) z=2 \times 10^{-2} \mathrm{~m} ;(d) z=3 \times 10^{-2} \mathrm{~m} ;(e) z=4 \times 10^{-2} \mathrm{~m}$ and $(f) z=5 \times 10^{-2} \mathrm{~m}$.

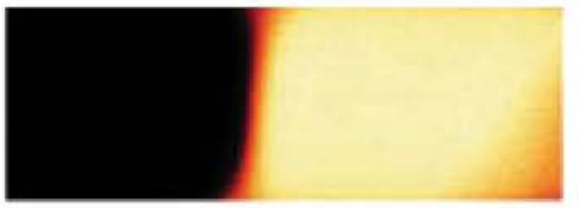

(a)

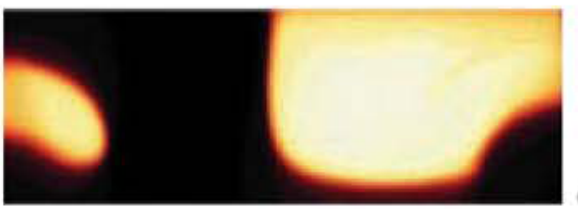

(b)

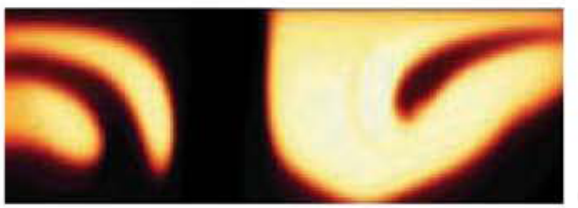

(c)

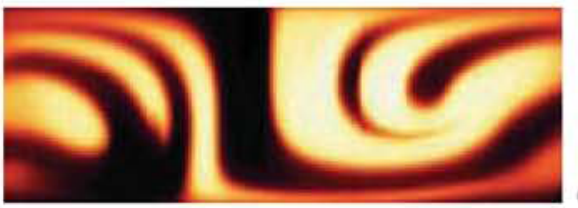

(d)

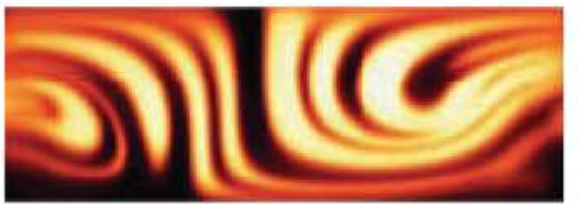

(e)

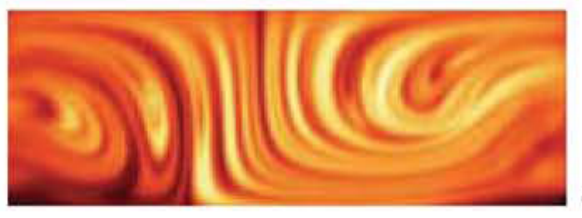

$(f)$

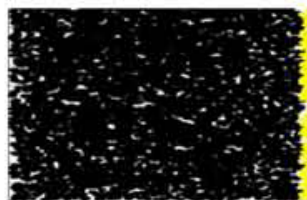

(a')
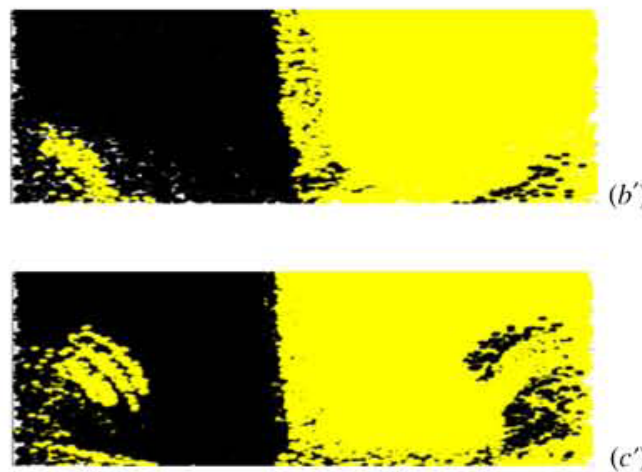

$\left(c^{\prime}\right)$
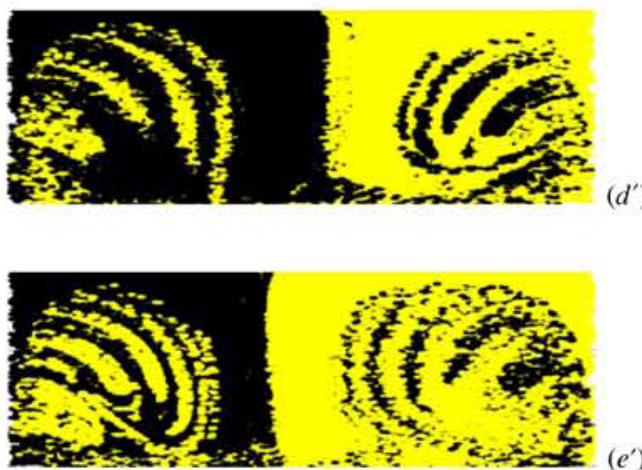

$\left(e^{\prime}\right)$

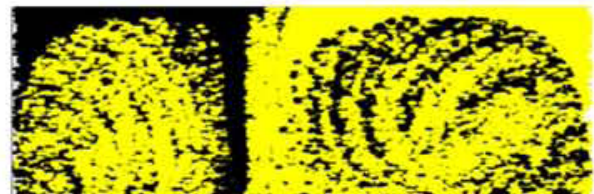

$\left(f^{\prime}\right)$

Figure 4. From $(a)$ to $(f)$ : evolution of mixing in the SHM micromixer; pictures form Stroock et al [10]. From $\left(a^{\prime}\right)$ to $\left(f^{\prime}\right)$ : results from 3D CFD simulations with 10000 tracers used to simulate the mixing of the two fluids. 
numerical simulations. To validate our numerical work we also analyze the mixing process in the staggered herringbone mixer (SHM) and compare it to the published experimental results [10].

The boundary conditions for the CFD simulations of the pressure-driven flow are an average velocity in the down channel direction of $1 \mathrm{~m} \mathrm{~s}^{-1}$ and no slip at the solid surfaces. The fluid is Newtonian with a density of $10^{3} \mathrm{~kg} \mathrm{~m}^{-3}$ and viscosity of $10^{-3} \mathrm{~Pa} \mathrm{~s}$. The finite element meshes comprise different number of elements for the different geometries: 103000 for the simple channel, 143000 for the straight ridge geometry and 246000 for the AVR geometry. The field equations for the 3D steady-state, isothermal flow were solved using FIDAP, employing an implicit time integration scheme and conjugate gradient-based iterative solvers with a convergence criterion of 0.01 . As explained above, 8000 tracers of two colors ( 4000 per species) are randomly placed at the inlet of the mixers to completely fill the cross-section, and the particle tracking algorithm (equation (5)) is used to calculate the tracers' trajectories and observe the dynamics of the mixing process.

The initial tracer distribution and progression of mixing of the two fluids are shown in figures 3 and 4 for the SDR micromixer and the AVR micromixer, respectively. There is no change between the inlet and exit configurations in the straight channel geometry, and we do not present this result graphically. A visual inspection of figures 3 and 4 provides a qualitative assessment of mixing in different geometries. And although formation of striations can be clearly observed for both the SDR and the AVR geometries, mixing seems to progress faster in the AVR system by comparison with the SDR geometry. Figure 4 also shows a side-by-side comparison of the results obtained in the experiments in [10] with our results from the CFD calculation.

\section{Results and discussion}

A study of the entropy $S$ (location) given in equation (4) confirms that this term can be neglected in the mixing analysis since it is always constant, as shown in figure 5 .

We quantify the level of mixing at different cross-sections along the channel length by employing $S_{\text {location }}$ (species) normalized by $\ln 2$, as explained above. The results of this analysis are presented in figure 6 . There is no mixing between the two species in the straight channel and consequently $S_{\text {location }}$ (species) remains nil along the channel length. Plotted in figure 6 are the normalized $S_{\text {location }}$ (species) for the SDR and AVR geometries only. One can clearly observe that the normalized $S_{\text {location }}$ (species) attains higher values in the AVR channel by comparison with the SDR geometry. Also, looking at the dynamics of the mixing process, one can distinguish a higher initial slope of the normalized $S_{\text {location }}$ (species) versus the channel length in the case of the AVR configuration by comparison with the SDR mixer.

Figure 7 also illustrates the effect of changing the number of bins, i.e., the scale of observation, in the mixing analysis. As the number of bins increases and their size decreases, analysis is done at a smaller scale of observation, and consequently species segregation becomes more apparent with an inherent decrease in entropy. However, the higher mixing efficiency of

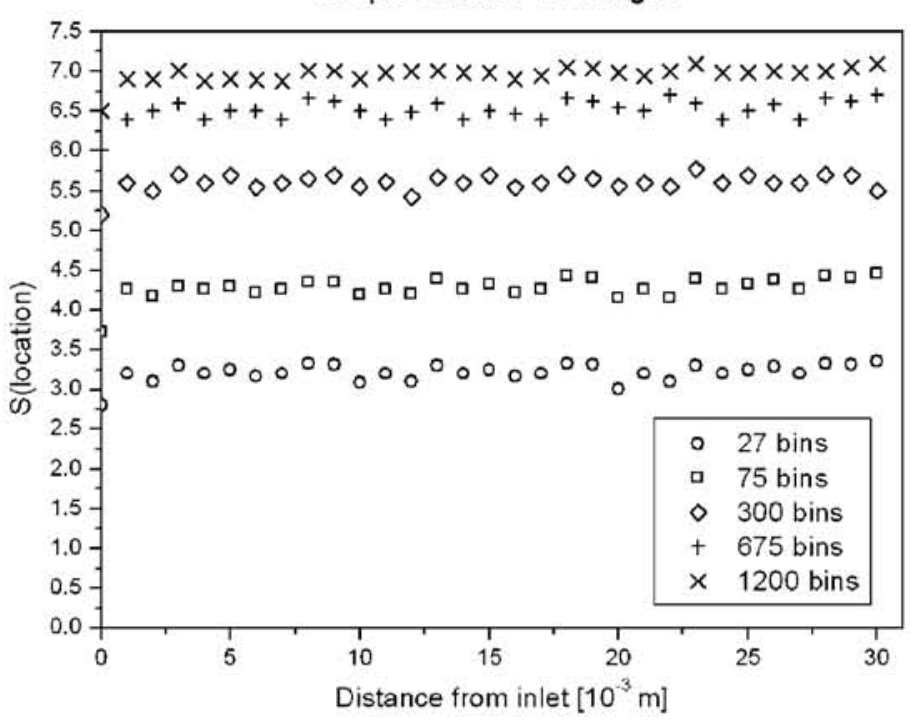

SDR Channel - Straight Diagonal Ridges

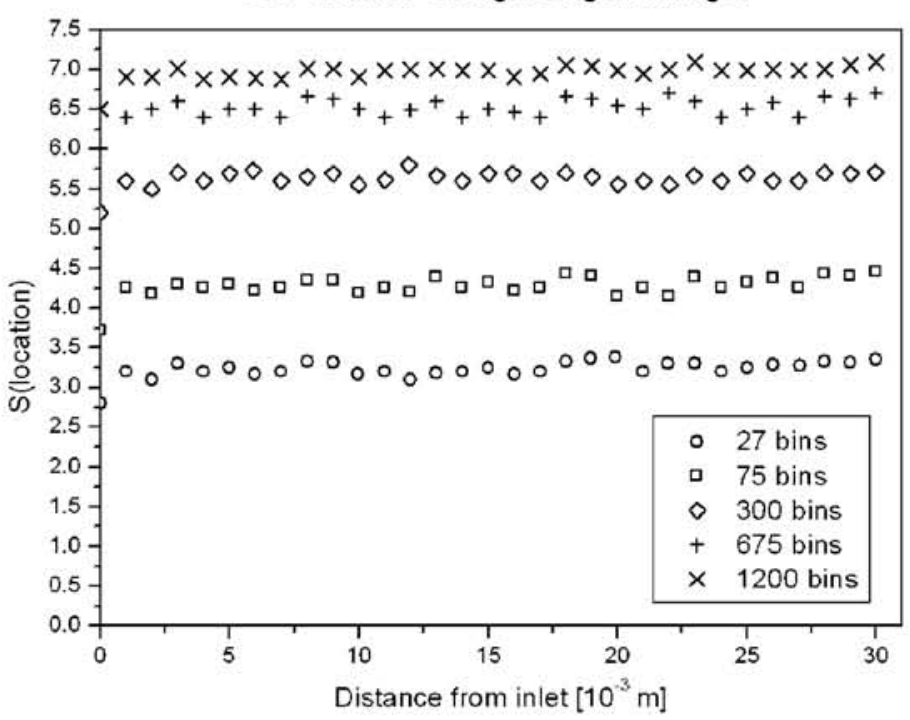

AVR Channel - Asymmetric V Ridges

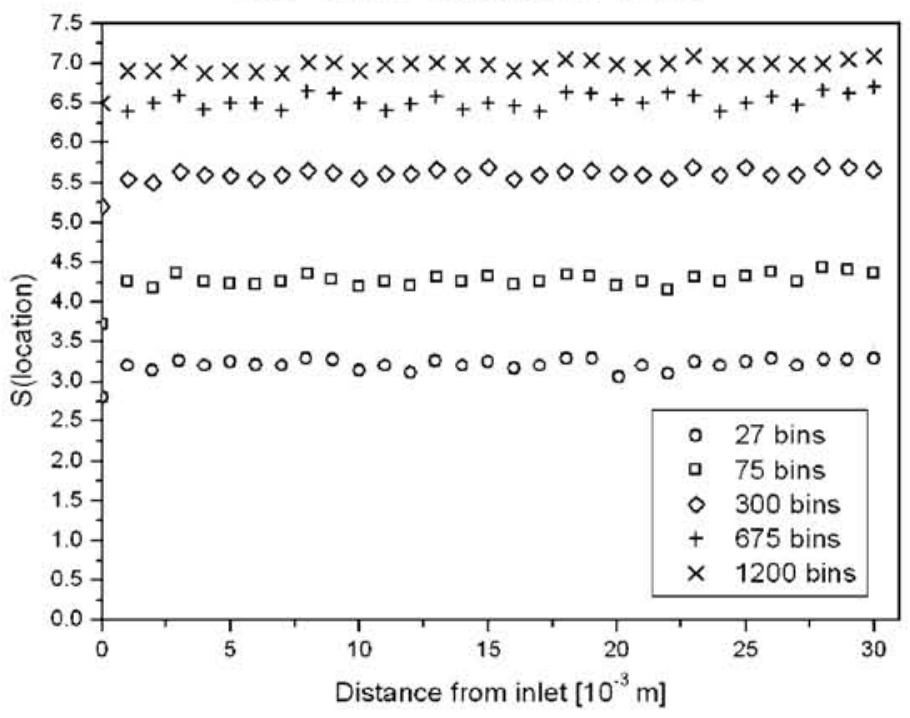

Figure 5. $S$ (location) versus distance from the inlet for different number of bins for the three systems. There is no dependence of the entropy $S$ (location) on the $z$ distance, therefore the mixing analysis can be done neglecting this term and using only the entropy $S_{\text {location }}$ (species).

the AVR configuration is apparent at all scales of observation. Also, when using a high number of bins, particularly in the 

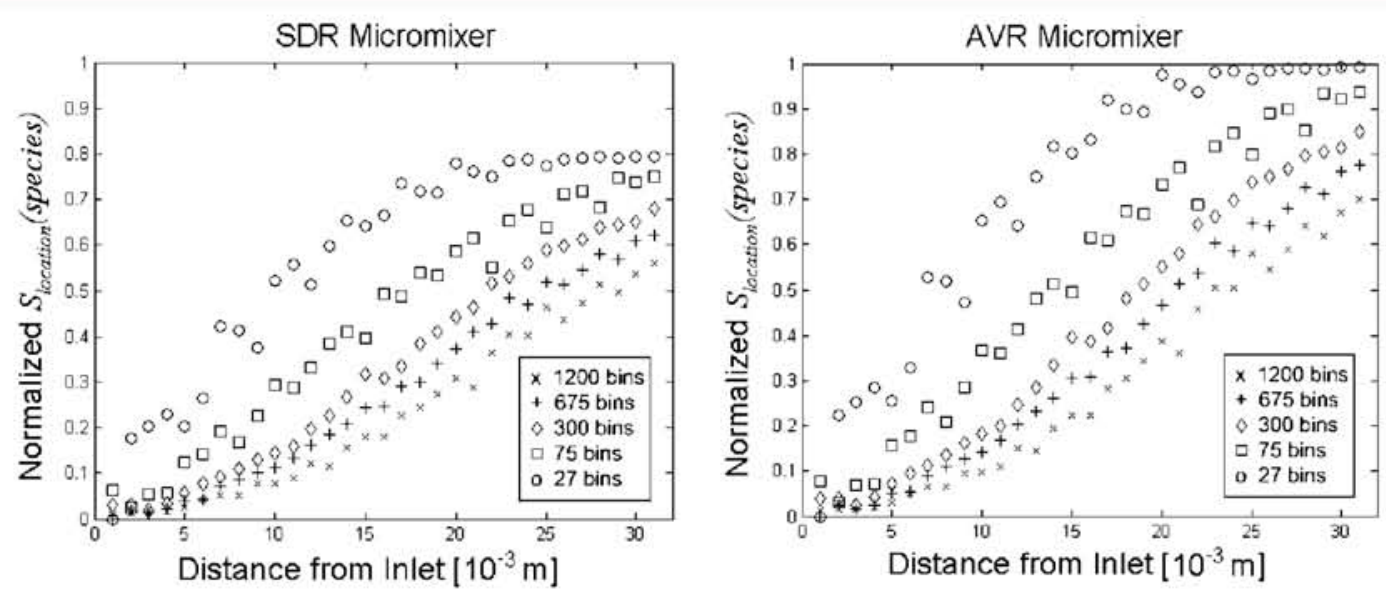

Figure 6. Normalized $S_{\text {location }}$ (species) versus distance from the inlet for different number of bins. The maximum values reached by the AVR mixer are higher by comparison with the SDR mixer. The effect of the number of bins used in the analysis is clearly visible.
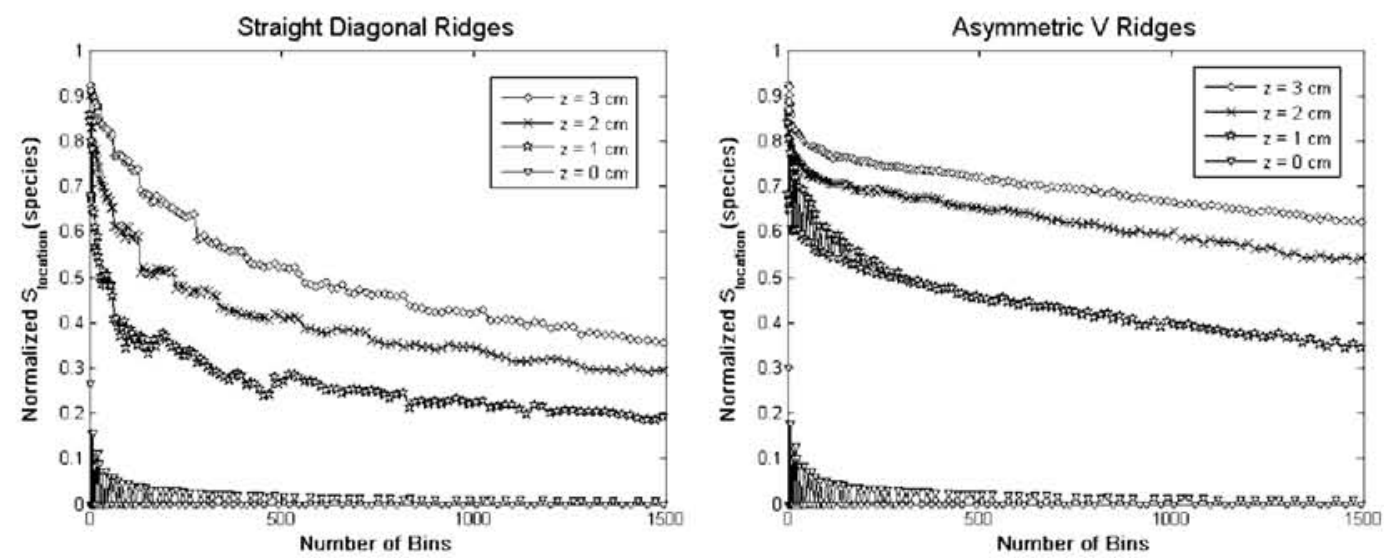

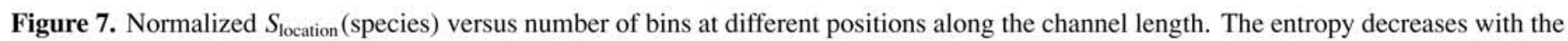
scale of observation, except for the inlet position. At various cross-sections along the channel, the decrease is more pronounced for the SDR system.

case of the AVR system, the analysis reflects the incomplete mixing of the species at the exit from the channel as evidenced in figure 5 .

A more detailed understanding of the effect of the scale of observation on the mixing quality can be obtained by plotting the normalized $S_{\text {location }}$ (species) versus the number of bins at different distances from the inlet. The results are shown in figure 7 and, besides the higher values of the normalized $S_{\text {location (species) for the case of the AVR system compared }}$ to the SDR one, we note that except for the initial crosssection, the normalized entropy decreases monotonically with the number of bins, suggesting that additional information on mixing/segregation can be obtained at smaller scales of observation. It is interesting to note that at large scales of observation, e.g., small number of bins employed, the decrease in entropy with the number of bins is more pronounced for the SDR system by comparison with the AVR one. This observation points out to more stringent differences in the level of mixing perceived at different scales of observation in the SDR system in contrast to the AVR geometry where mixing appears to be perceived more alike. This observation calls for a further investigation. The computational time in this analysis (entropy computed for 30 cross-sections using between 27 and 1500 bins) is on the order of minutes on a regular PC, varying, although not significantly, with the number of bins used.

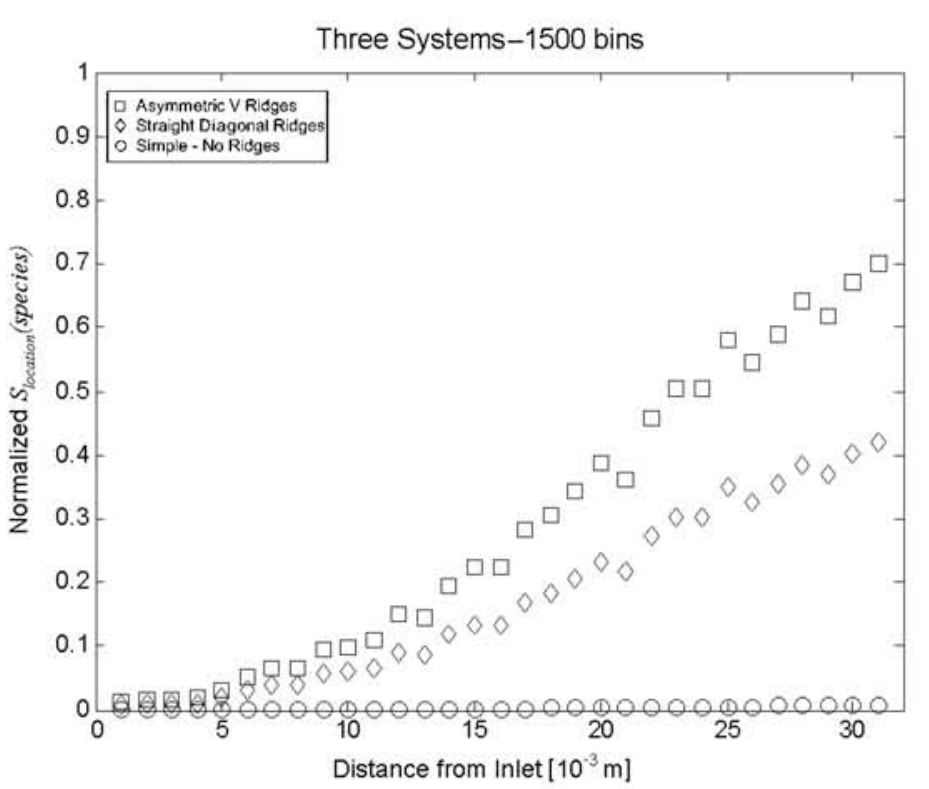

Figure 8. Comparison of mixing efficiency in the three different geometries.

To facilitate comparison of the three systems, in figure 8 we plot the normalized $S_{\text {location }}$ (species) versus the distance from the inlet for the three different geometries at 1500 bins, the highest level of resolution/smallest scale of observation used in the analysis. The AVR mixer clearly shows the highest 


\section{Entropic Analysis of Pictures}

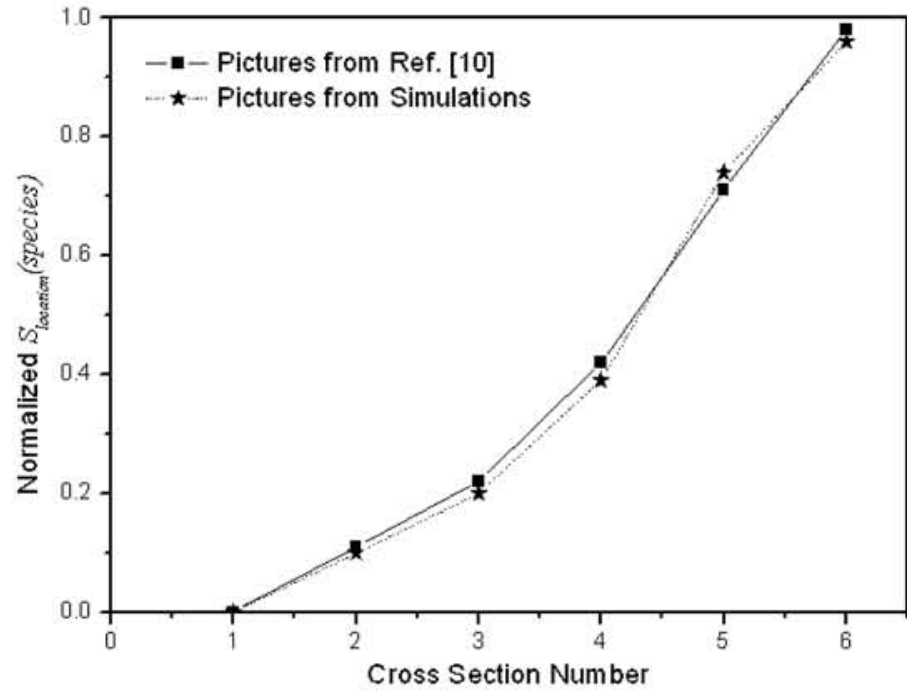

Figure 9. Comparison of the entropic analyses of the pictures presented in figure 4 . The pictures are analyzed based on the grayscale value in each pixel as converted from figure 4. Every picture is divided into 1500 bins and the entropy $S_{\text {location }}$ (species) is calculated based on the probabilities $p_{1 / j}$ and $p_{2 / j}$ representing the averages over the total number of pixels in bin $j$ for the black and white levels, respectively.

mixing efficiency both in terms of mixing quality and mixing rate.

We finally apply our entropic analysis on the experimental results obtained by Stroock et al [10] in the staggered herringbone mixer (SHM). In figure 4 we show six pictures given in [10]. The qualitative feeling that the mixing of the two fluids progresses from the top slice to the bottom one is confirmed quantitatively by the monotonic increase of $S_{\text {location }}$ (species) $/ \ln (2)$ shown in figure 9 . To compute the entropy, each of the six cross-sections has been converted to gray scale, divided into 1500 bins, and $S_{\text {location }}$ (species) was computed from equations (2) and (3). The same procedure has been also applied for the pictures obtained from the numerical simulation of the AVR, see figure 4, and the conditional entropy is also shown in figure 9. The result of the entropic analysis is gratifying as it demonstrates that the methodology proposed here can be successfully applied to both numerical simulations and experiments.

\section{Conclusions}

In this work we have shown how to implement a rigorous quantitative assessment of mixing quality in microfluidic devices, through the use of the Shannon entropy. The devices we analyzed in this paper have geometries similar to, although not identical with the ones presented by Stroock et al [10]. Our results show an enhanced mixing efficiency for the AVR system (a similar version of the SHM mixer) by comparison with the SDR system. We have shown no mixing of the two fluids in the non-patterned channel, a result also verified experimentally. Our rigorous analysis of mixing opens the way for virtual processing aiming at design optimization of microchannels. For example, as demonstrated in figures 6 and 8, the mixing achieved in an AVR mixer of length $2 \times$ $10^{-2} \mathrm{~m}$ is quite close to the maximum, thus building a longer mixer will not result in better mixing. One can infer from this result an optimum length of $2 \times 10^{-2} \mathrm{~m}$ for this mixer. We have also analyzed directly the experimental data from the SHM mixer ${ }^{10}$ demonstrating the versatility of the entropic method which can be applied to both simulations and experiments.

\section{Acknowledgment}

The authors thank the National Science Foundation for the financial support for this research through grant DMI0140412 .

\section{References}

[1] Burns M A et al 1998 Science 282 484-96

[2] Dunn D A and Feygin I 2000 Drug Discov. Today 12 (Suppl.) S84

[3] Losey M W, Schmidt M A and Jensen K F 2001 Ind. Eng. Chem. Res. 402555

[4] Sammarco T S and Burns M A 1999 AIChE J. 45350

[5] Moore S K 2001 IEEE Spectr. 38 28-9

[6] Jones S W and Young W R 1994 J. Fluid Mech. 280149

[7] Pfahler J, Harley J, Bau H and Zemel J 1990 Sensors Actuators A 23 431-4

[8] Jendrejack R M, dePablo J J and Graham M D 2002 J. Chem. Phys. 116 7752-9

[9] Ottino J M 1989 The Kinematics of Mixing: Stretching, Chaos and Transport (Cambridge: Cambridge University Press)

[10] Stroock A D, Dertinger S K W, Ajdari A, Mezic I, Stone H A and Whitesides G M 2002 Science 295 647-51

[11] Liu R H, Stremler M A, Sharp K V, Olsen M G, Santiago J G, Adrian R J, Aref H and Beebe D J $2000 \mathrm{~J}$. Microelectromech. Syst. 2 190-7

[12] Therriault D, White S R and Lewis J A 2003 Nature Mater: 2 265-71

[13] Yamaguchi Y, Takagi F, Watari T, Yamashita K, Nakamura H, Shimizu H and Maeda H 2004 Chem. Eng. J. 101 367-72

[14] Wang H, Iovenitti P, Harvey E and Masood S 2003 J. Micromech. Microeng. 13 801-8

[15] Niu X and Lee Y 2003 J. Micromech. Microeng. 13 454-62

[16] Devaney R 1989 An Introduction to Chaotic Dynamical Systems (New York: Addison-Wesley)

[17] Shannon C E 1948 The Bell Syst. Tech. J. 27 379-423

Shannon C E 1948 The Bell Syst. Tech. J. 27 623-56

[18] Brooks D R 1998 Evolution as Entropy (Chicago: University of Chicago Press)

[19] Allen P A, Kaufman M, Smith A F and Propper R E 1998 Psychol. Aging 13 501-18

[20] Georgescu-Roegen N 1971 The Entropy Law and the Economic Process (Cambridge, MA: Harvard University Press)

[21] D'Allessandro D, Dahleh M and Mezic I 1999 IEEE Trans. Autom. Control 441852

[22] Khinchin A I 1957 Mathematical Foundations of Information Theory (New York: Dover)

[23] Wang W, Manas-Zloczower I and Kaufman M 2001 Intern. Polym. Process. 161

[24] Wang W, Manas-Zloczower I and Kaufman M 2003 AIChE J. 491644

[25] Alemaskin K, Camesasca M, Manas-Zloczower I, Kaufman M, Kim E K, Spalding M, Trumbull W and Swain R 2004 ANTEC 2004 Conf. Proc.

[26] Kang T G and Kwon T H 2004 J. Micromech. Microeng. 14 891-9

[27] Reddy J N and Gartling D K 2000 The Finite Element Method in Heat Transfer and Fluid Dynamics 2nd edn (Boca Raton, FL: CRC Press)

[28] Anderson J D Jr 1995 Computational Fluid Dynamics: the Basics with Applications (New York: McGraw-Hill) 